\title{
Routing Optimization for Delay Tolerant Networks in Rural Applications Using a Distributed Algorithm
}

\author{
C. Velásquez-Villada, F. Solano, Y. Donoso
}

\author{
Carlos Velásquez-Villada \\ Universidad de los Andes \\ Bogotá D.C., Colombia, South America \\ c.velasquez@ieee.org \\ Fernando Solano \\ Warsaw University of Technology \\ Poland, Nowowiejska 15/19, Warsaw \\ fs@tele.pw.edu.pl \\ Yezid Donoso* \\ Universidad de los Andes \\ Bogotá D.C., Colombia, South America \\ *Corresponding author: ydonoso@uniandes.edu.co
}

\begin{abstract}
Internet access can improve people's life quality by helping them to reduce and overcome the poverty and educational gaps. However, most rural communities in the world, specially in underdeveloped countries, do not have access to the Internet. Delay/Disruption Tolerant Networking (DTN) is a recent low-cost technology now being used to provide connectivity to rural towns were some transportation means periodically arrive. DTNs can be implemented to connect communities to Internet, since this technology takes advantage of the existing people's transportation infrastructure using it to move packets and messages to and from Internet.

This paper proposes a DTN mathematical optimization model that maximizes the availability probabilities of the paths from sources to destinations. We also present an opportunistic forwarding algorithm that takes into account the availability probability of a node's neighbors to decide if a node should forward a message or store the message until a node with a higher availability probability contacts it. This algorithm was tested in five different scenarios and in all of them it found a path to the destination. Keywords: Disruption-Tolerant, Delay-Tolerant, availability probability, opportunistic forwarding, ICT for rural development, Rural telecommunications.
\end{abstract}

\section{Introduction}

Most rural communities in the world have scarce or non-existent Internet availability [1]. According to the ITU [1], only $38.8 \%$ of the world's population have access to Internet, either fixed or mobile. Most of these connections are concentrated in the main cities, leaving most of the world's surface with almost no Internet availability. Several governments are increasing the availability of Internet in their territories, showing an increase of $145.57 \%$ from 2006 to 2013 , as can be seen in the Internet penetration figures from ITU [1]. The initiatives to bring Internet to more people include optical fiber deployments, satellite connectivity solutions, electronic devices for people in remote towns and training for them to use these new devices and connections.

Even with these efforts, many people will still be left behind without constant Internet connectivity or without connectivity at all. The Delay/Disruption Tolerant Networking (DTN) architecture proposed in [2] is a new technology that can connect users in rural and disconnected places. This DTN technology can give disconnected users access to information that can help them overcome the digital divide and become net citizens taking full advantage of the information, 
education and opportunities that Internet can provide. Benefits of this connectivity include access to learning materials like Wikipedia, distance learning courses or even offline versions of Massive Online Open Courses (MOOCs) that gives them the same education contents that their urban counterparts receive; thus, helping them to reduce the poverty gap $[1,3,4]$.

There are also benefits when the local infrastructure is created in the communities where people can create contents and share them with others. Local news can be reported on a local website, people can share their music, documents, videos or libraries. In some communities, people have created local websites for their businesses and even local radio stations over the net. These applications depend on a strong deployment of a local infrastructure and the presence of people willing to train and educate locals. Even without the community network, the opportunities that can arise from a connection point and information distribution in an asynchronous way can bring many benefits for the inhabitants around these deployments. Applications that show the benefits of giving ICT to people in remote communities and accompanying these technologies with the respective knowledge, training and following can be found in many fields: Healthcare [5-7], banking opportunities [8,9], agriculture development [10-12], businesses creation and growing [8,13], politics accountability and participation [14], and education [15].

Although there are many studies about DTN architectures and technologies (as can be seen in Section 2), research and developments in application software and specially in routing protocols are still missing. Most current applications and protocols fail when implemented in a DTN environment, due to long delays or frequent disruptions. The focus of this work is on developing a routing algorithm that can be used in a real application to bring Internet connectivity to rural communities around the world.

This paper introduces a mathematical model to represent the best path choices in a Delay/Disruption Tolerant network designed for rural connectivity scenarios. This model is built based on previous models developed by the authors [28-30]. The model uses the availability probabilities of the network's links and their changes through time to calculate the best paths from the information sources to their destination. The model gives, as a result, the optimal decisions that the nodes can make to send their information to the destination (when should a node send a message to a neighbor and to which neighbor and when should this node store the message in its buffer to send it later instead). This paper also introduces a distributed heuristic algorithm to make forwarding decisions. This algorithm is implemented at each node in the simulation, and it decides whether to forward or store the messages based on the availability probability of a node's neighbors (if this availability probability is good enough will be decided via a variable parameter that will be used and tested in the simulation).

This paper extends paper [31]. The key additions of this journal version are as follows. First, Section 2 includes and describes more related works, the DTN Architecture, and DTN implementations for rural solutions. Secondly, this paper contains an extended explanation of the mathematical model presented in Section 3 and of the heuristic algorithm from Section 4. Finally, this paper contains additional results for the simulated scenarios.

This paper is organized as follows: Previous and related works are summarized in Section 2; Section 3 introduces the mathematical model used to calculate the optimal forwarding decisions; Section 4 describes the distributed heuristic algorithm implemented for the simulation; Section 5 shows and discusses optimization and simulation results; finally, conclusions and possible directions for future research are summarized at the end of the document.

\section{Related Work and DTN Architecture}

Delay/Disruption Tolerant Networks (DTN) are a relatively new kind of network that work in challenging environments $[2,16-23]$. These networks have been developed with new protocols 
tailored to their needs, which can make them incompatible between different applications and even with the Internet. Each one of these networks works within its own boundaries with known and relatively homogeneous delays and error rates, and some have trusted boundary devices to translate inner messages to different protocols for external communications.

The initial application for the DTN, where the name appeared, was the Interplanetary Internet Project [17], an idea from NASA to interconnect devices in space. It was motivated from the Pathfinder mission to Mars (1997), but it was tested in the Spirit and Opportunity missions in Mars (2004) due to a failure in the communication solution implemented in one of these rovers. After this experience, NASA started working and researching for an InterPlanetary Internet with the idea of connecting most of the devices in the space to provide a reliable store and forward network for all current and future missions. They also imagined a future where different spatial agencies could use these protocols and extend the reach of this InterPlanetary Network (IPN) for further spatial exploration.

Earthly DTN protocols face other challenges, mainly high rates of disruptions or failures. They must enable interconnectivity between different networking technologies in order to allow these technologies-used for wireless sensor networks, unmanned vehicular networks, battlefield monitoring and smart infrastructure-to communicate through the Internet and with each other. Protocols must account for variable delays, variable error rates, network disruptions and attacks.

The architecture for DTN proposed in [2] tries to change or at least relax some assumptions built into the Internet architecture. These assumptions are that there are end-to-end paths between source and destination; that retransmissions based on feedback from data receivers are effective for errors correction; that end-to-end loss is relatively small; that all network nodes support the TCP/IP protocols; that applications does not need to be aware of the communications performance; that security can be achieved through mechanisms on end nodes; that packet switching is the most appropriate abstraction for interoperability and performance; and, that a single route is sufficient for acceptable performance. The DTN architecture relaxes most of these assumptions using variable-length messages; a naming syntax that supports a wide range of naming and addressing; store-and-forward over multiple paths; security mechanisms against unauthorized use; classes of service; delivery options; and a way to express the useful lifetime of data.

The DTN architecture [2] uses a Bundle layer [21] that serves as middleware between the application layer and lower layers. This bundle layer shows a transparent interface for applications that can go through the Internet or DTN. However, applications for DTN must follow some design principles: they should minimize the number of round-trip exchanges, cope with restarts after failure while network transactions remain pending, and inform the network of the useful life and relative importance of data to be delivered. The bundle layer provides unacknowledged, prioritized (but not guaranteed) unicast message delivery. It also provides two options for enhancing delivery reliability: end-to-end acknowledgments and custody transfer. Applications can use these end-to-end acknowledgments for reliability. The custody transfer option allows a node to give the responsibility for reliable transfer of messages to other node, a "custodian". Nodes in the DTN can be custodians or can choose not to be based on available resources and network congestion. A custodian-to-custodian acknowledgement mechanism is implemented in the bundle layer.

The Bundle Protocol defines a convergence layer where underlying protocols will function to provide successful end-to-end communications. These convergence layers accomplish communications between nodes and can comprise a whole network stack with transport and network protocols that can run on top of existing networking technologies. Many protocols can exist in these convergence layers (the protocols themselves are called convergence layers), but they must provide at least two services to the bundle protocol agent: "sending a bundle to all bundle 
nodes in the minimum reception group of the endpoint identified by a specified endpoint ID that are reachable via the convergence layer protocol; and delivering to the bundle protocol agent a bundle that was sent by a remote bundle node via the convergence layer protocol." [21].

Demmer [24] presents a full implementation of the previous architecture with storage and routing developments and an available simulation environment. Daknet [25] is a project developed by MIT researchers that tackles the problem of Internet connectivity in rural or remote communities and is the closest one to the idea presented here. Daknet aims to provide connectivity to remote villages were some wireless networking devices have been installed (kiosks) in strategic places and in buses or public service vehicles (Mobile Access Points, MAPs) that eventually will go to this and other villages and bigger towns, delivering messages between these disconnected networks and also accessing Internet (for non-real time access) at some moment and delivering and retrieving requested information. Authors in [20], [26] and [27] present developments on DTNs for underdeveloped regions concluding that providing full reliability and good strategies for data forwarding are difficult objectives in these scenarios.

\section{Optimization Problem}

Topology of DTNs is usually unreliable and constantly changes due to their nodes movement. However, we can use these same node movements to transmit messages in the network and to Internet. In this section, we present the problem of finding the best path in the network from one or more sources to a destination (it can be a node connected to Internet) based on the availability probabilities of the links between the nodes. The mathematical model takes into account that the availability probability of a link can change from one instant of time to another. The model has complete information over the network and can decide whether to send a message over a link or wait (storing the message in an internal buffer) until there is a link with a better availability probability. Thus, the network model presented in this paper maximizes the availability probability of the paths from sources to destinations, see Fig. 1. The model assumes that every node has storage capabilities, that it can serve as a relay for messages from other nodes and that it can send a message at any moment of time. The links between the nodes are given as are given their availability probabilities that serve to model the movement of the nodes, these probabilities are assumed to be independent and following an uniform distribution for testing purposes.

Table 1: Mathematical model parameters

\begin{tabular}{ll}
\hline Parameter & Definition \\
\hline$P$ & Set of candidate paths \\
$T$ & Set of discrete time intervals \\
$c_{i j}$ & Capacity of edge $(i, j) \in E$. \\
$c_{i i}$ & Storage capacity of node $i$ \\
$a_{i j}(t)$ & Availability Probability of edge $(i, j) \in E$ at time $t$ \\
& It is assumed that the availability probability of the edge $(i, j)$ \\
& is the same as the one for the edge $(j, i)$ \\
$t$ & discrete time interval, where $t \in T$ \\
$\delta_{t}$ & Time interval duration \\
\hline
\end{tabular}

The model starts with a network represented by a graph $G=(N, E)$, where $N$ is the nodes set and $E$ is the edges set. The edges set has the connections between the nodes. The graph $G$ is extended in a new graph $G^{\prime}=\left(N^{\prime}, E \prime\right)$ that contains a copy of the original graph for every change 


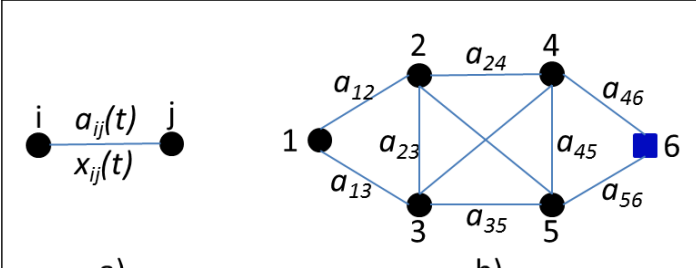

a)

b)

Figure 1: Network representation (a) two-node graph; (b) six-node graph

in a link's availability probability. The extended graph $G$ ' is used by the optimization algorithm to make decisions on forwarding or storing the messages, since it has all the information about the network changes, see Fig. 2. Table 1 shows the main parameters used in the mathematical model. Set $T$ contains the time intervals $t$ that define the validity of the links' availability probabilities. These time intervals have a duration equal to $\delta_{t}$. We are assuming that all time intervals have the same extent and that this time period lasts long enough to send a message between two neighboring nodes. Capacities are assumed to be constant, since they depend on the hardware of the nodes and because of this are not so easily changed.

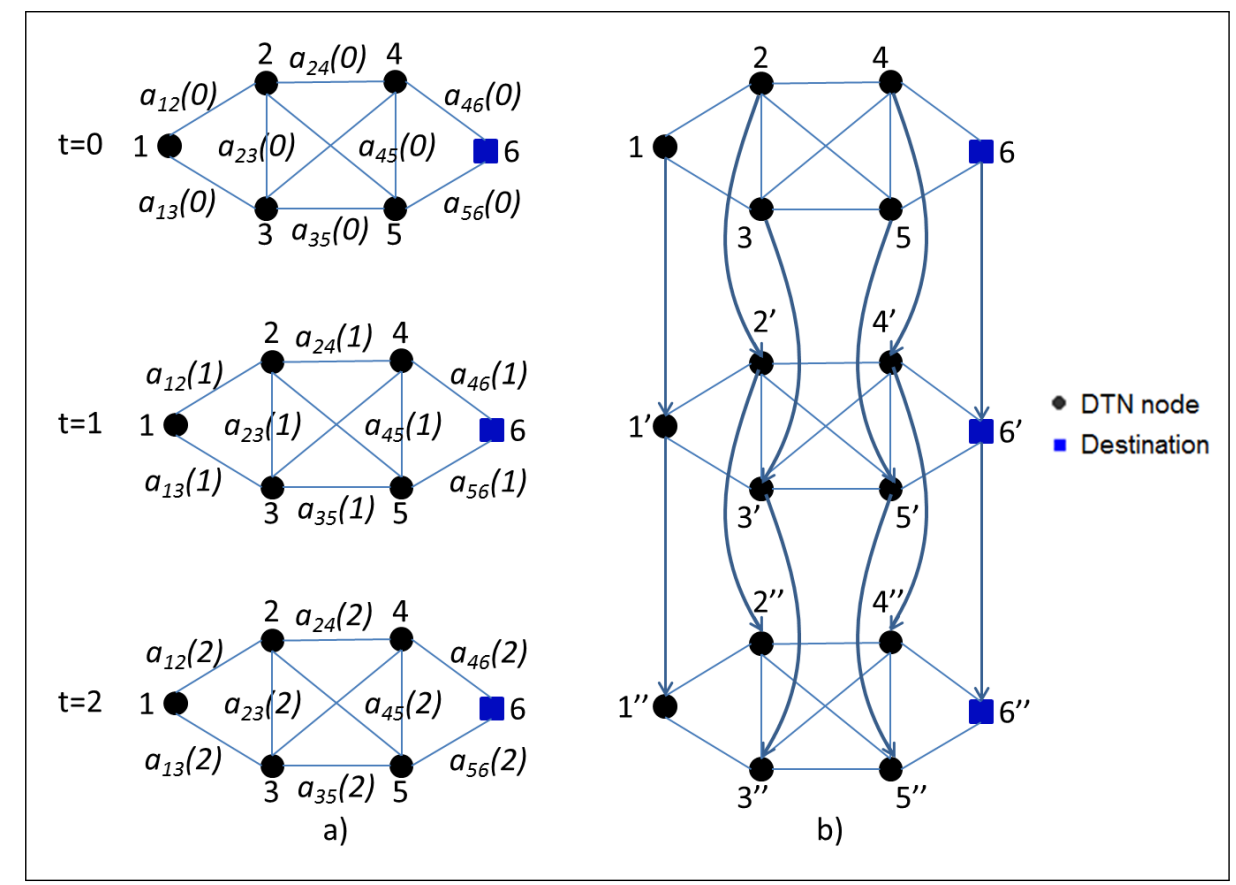

Figure 2: Network graph (a) original graph changes through time; (b) extended graph with the availability probabilities through time represented at once

$$
\begin{array}{r}
\max \prod_{(i, j) \in P} a_{i j}(t) x_{i j}(t) \\
\max \sum_{(i, j) \in E} \log \left(a_{i j}(t)\right) x_{i j}(t)
\end{array}
$$


Constraints

$$
\begin{gathered}
\sum_{j \in N} x_{i j}(t)-\sum_{j \in N} x_{j i}(t)+x_{i i}(t)-x_{i i}(t-1)=b_{i}(t) \quad \forall(i, j) \in E, i \neq j \\
x_{i j}(t) \leq c_{i j} \delta_{t} \quad \forall(i, j) \in E \\
x_{i j}(t) \in \mathbb{Z}_{\geq 0} \quad \forall(i, j) \in E, p \in P
\end{gathered}
$$

Equation (1) shows the objective function that maximizes the network availability probability of the paths from sources to destination. This objective function multiplies the availability probabilities of the links in a path, since we are dealing with probabilities and we want to obtain the path that maximizes these availability probabilities. We are assuming that the internal buffer is always available, so this availability probability (to store a message in its buffer) is always 1 . A linear approximation of the objective function is shown in Equation (2). Decision variable $x_{i j}(t)$ determines the amount of information that flows through the link $(i, j)$ using positive integers (5). $x_{i j}(t)$ is greater or equal than 1 if the link $(i, j)$ is in the path $p$ for a source to a destination, and 0 otherwise.

Constraints (3) and (4) are data-flow constraints and they keep the information flow below the channels and buffers capacities and guarantee that the messages reach their destinations.

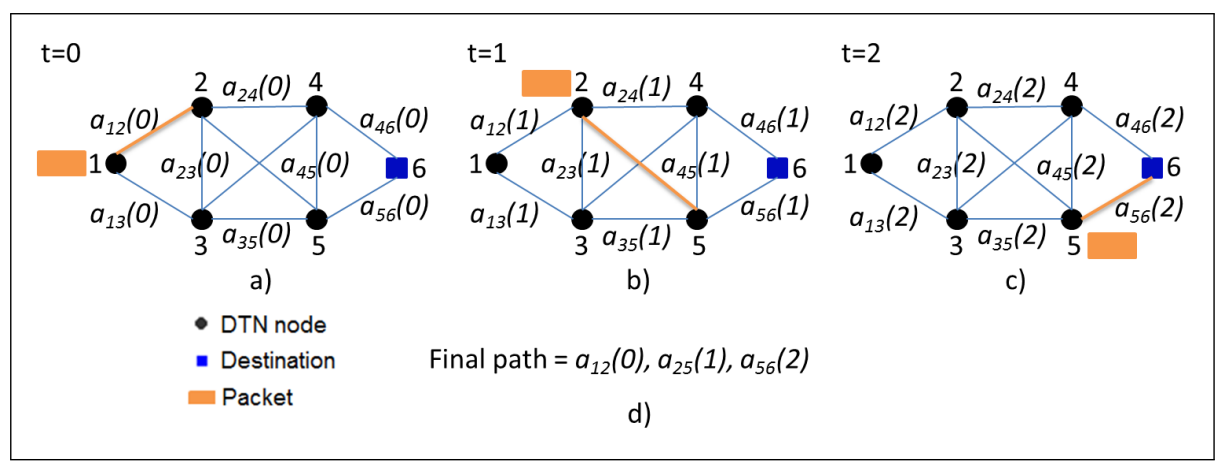

Figure 3: Network routing solution (a) decision taken at time 0; (b) decision taken at time 1; (c) decision taken at time 2 ; (d) final path through time

Fig. 3 shows an example of the optimization algorithm at work. At time 0 node 1 creates a message to be sent to node 6 and all availability probabilities have their initial values. At this moment, node 1 chooses the highest availability probability to its neighbors and decides to send the message to node 2 . Then, at time 1 , all availability probabilities can change and node 2 have to decide where it should send the message or if the message should be stored in its internal buffer to be sent later. Node 2 decides to send the message to its neighbor with the highest availability probability, Node 5 . Finally, node 5 sends the message towards its destination, node 6 , at time 2 .

\section{Proposed Forwarding Heuristic}

This section describes the forwarding heuristic implemented for the results section based on the mathematical optimization algorithm. The heuristic internal working is explained in Fig. 4 and the pseudocode is shown in Algorithm 1. The heuristic works as a distributed optimization algorithm in which each node has to decide by its own and with local information whether it can send a message to a neighbor or it should store the message in its local buffer until a better path becomes available, see Fig. 4. For a node running the heuristic to decide if a link has a 
good enough availability probability it has to compare it against a set parameter (alpha). This forwarding heuristic can be used in a rural scenario that has at least one central, fixed wireless distribution point (AP), at least one mobile distribution point (MAP) that can be in contact with the local APs periodically (once a day or less in many cases, depending on the transportation means to the town). The APs and MAPs must have reasonable storage capabilities. The scenario has users in each village where at least an AP is deployed and users requests for information are not real-time ones and can wait for one or more MAP-AP contacts to be satisfied. Users must have the necessary technology to access the network and the appropriate training to use that technology and the applications given to them.

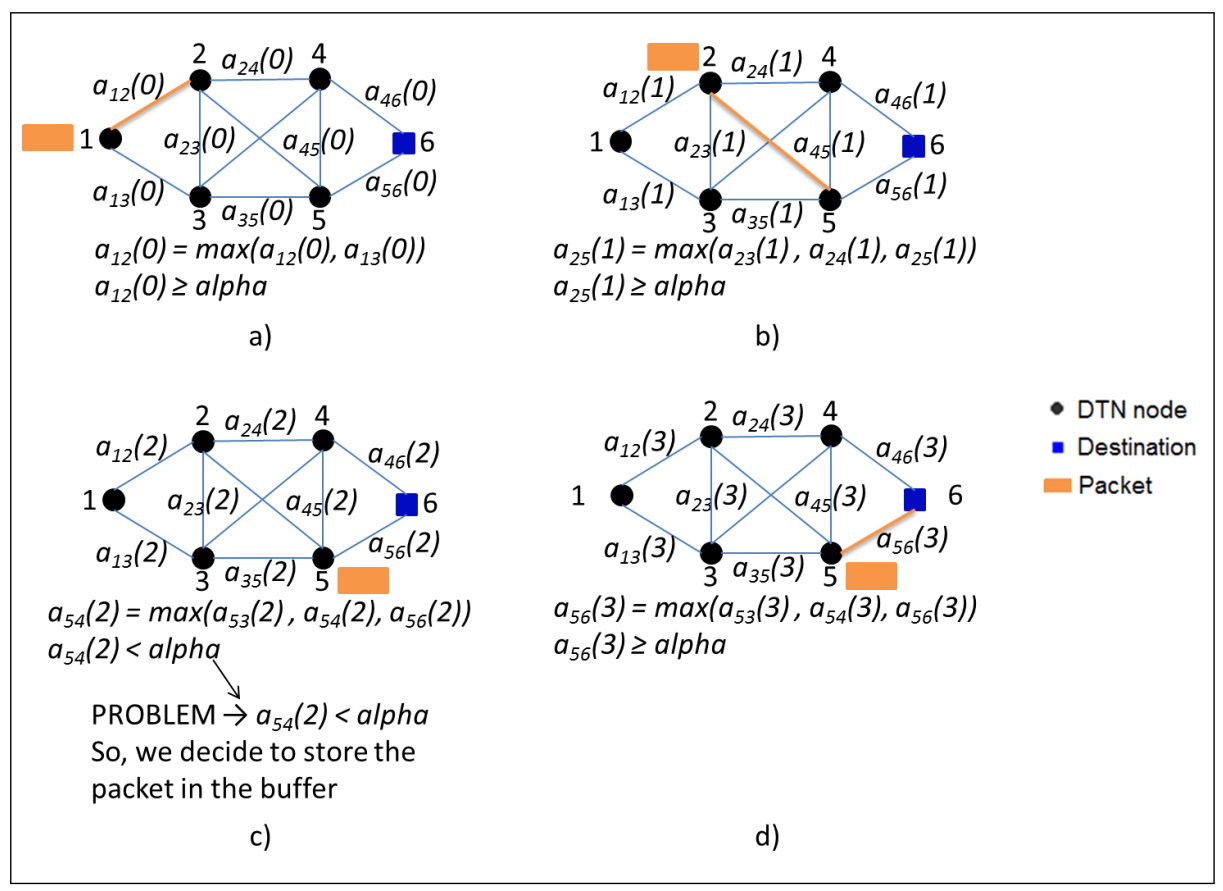

Figure 4: Network distributed heuristic (a) first step at time 0; (b) time 1 (c) time 2 (d) time 3

The forwarding algorithm, shown in Algorithm 1, is based on the assumption that every node knows the availability probability of its neighbors at any instant of time, and that if that probability is high enough (over a certain alpha) it can communicate with such neighbor. The alpha parameter is the threshold to take a forwarding action. If an availability probability is below this threshold (alpha) the node can assume that its neighbor is unreachable. Nodes do not have access to the whole network graph but they can discover which one is the destination or gateway in the network in order to send their requests to Internet. Nodes can create a request at any instant of time. In our forwarding strategy we take into account the information available at the node that is taking the forwarding decision; i.e., the availability probabilities of its neighbors. And we also take into account that the node should not return the packet to the node that sent it to him (but it can send it to another node, even if the packet visited that other node earlier).

\section{Results}

The proposed heuristic described in Section 4 was implemented using Java in a 64-bit CPU. Five different network configurations, from 6 to 10 nodes, as can be seen in Fig. 5, were simulated. In each scenario the last node received messages from all the previous nodes. These nodes created a message to be delivered through the path with the best availability probability but using only 


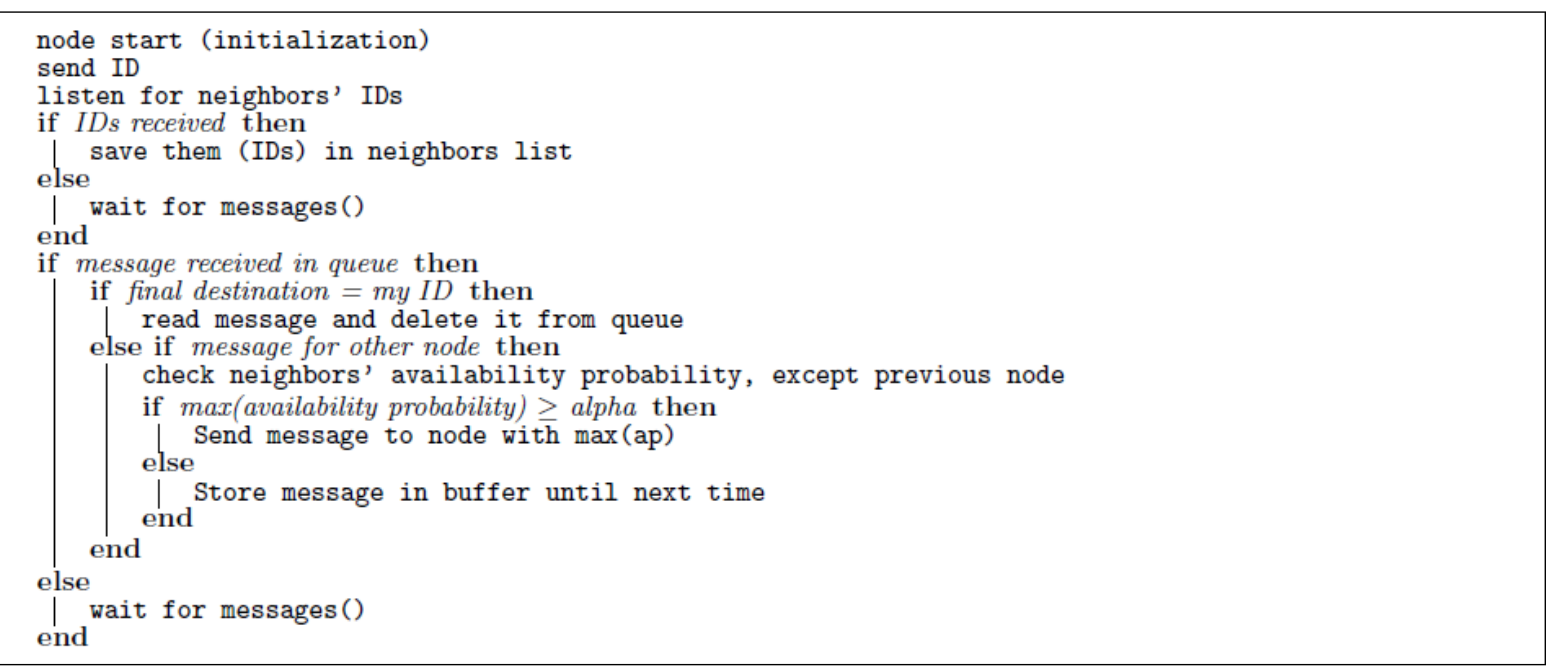

Algorithm 1: Distributed algorithm for every node in the network

local information. All nodes served as relays to convey messages to the destination (i.e., to the final node). Nodes had access to the availability probabilities of their links to their neighbors and they had to make a decision (send or store a message) based on this information and the parameter alpha (the threshold to decide if an availability probability is good enough). alpha was varied from 0.1 to 0.9 with 0.1 increases between each simulation run. We ran 10,000 simulations for each scenario and for every alpha to increase the confidence in the averaged results. The results, shown in Fig. 6, included the averaged values of all the availability probabilities of the paths from sources to destinations; the hop count and the time steps count until the last message was delivered to the final node. All these variables were measured for every alpha and plotted in a single graph for each scenario shown in Fig. 5 (see Fig. 6).

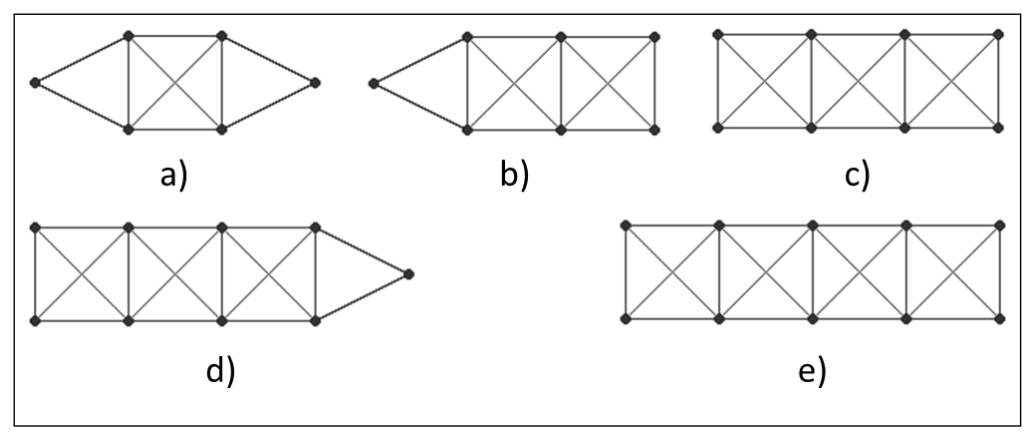

Figure 5: Scenarios used for the simulations with (a) 6; (b) 7; (c) 8; (d) 9; e) 10 nodes

As can be seen in Fig. 6, for alpha values between 0.1 and 0.6 there are no significant differences in all variables. The average hop count does not change; the time to deliver the messages presents an increase of around 7 steps (or a 30\% increase); and the average availability probability shows an increase of around 0.1 (or a $30 \%$ increase). For alpha values from 0.7 to 0.9 the hop count remains steady but the time steps and the availability probability increase exponentially. The change in time steps from 0.6 to 0.9 alpha is around $200 \%$; and the increase in availability probability is around 0.3 (an increase of around $77 \%$ ). These results show that choosing an adequate alpha value is a critical decision and that choosing an alpha between 0.6 and 0.8 most likely provides the best trade-off between time to deliver the messages and availability probability of the paths. From Fig. 6 we can also see that the hop count does not change with alpha variations and that it depends on the network size (number of nodes) and 
network topology.

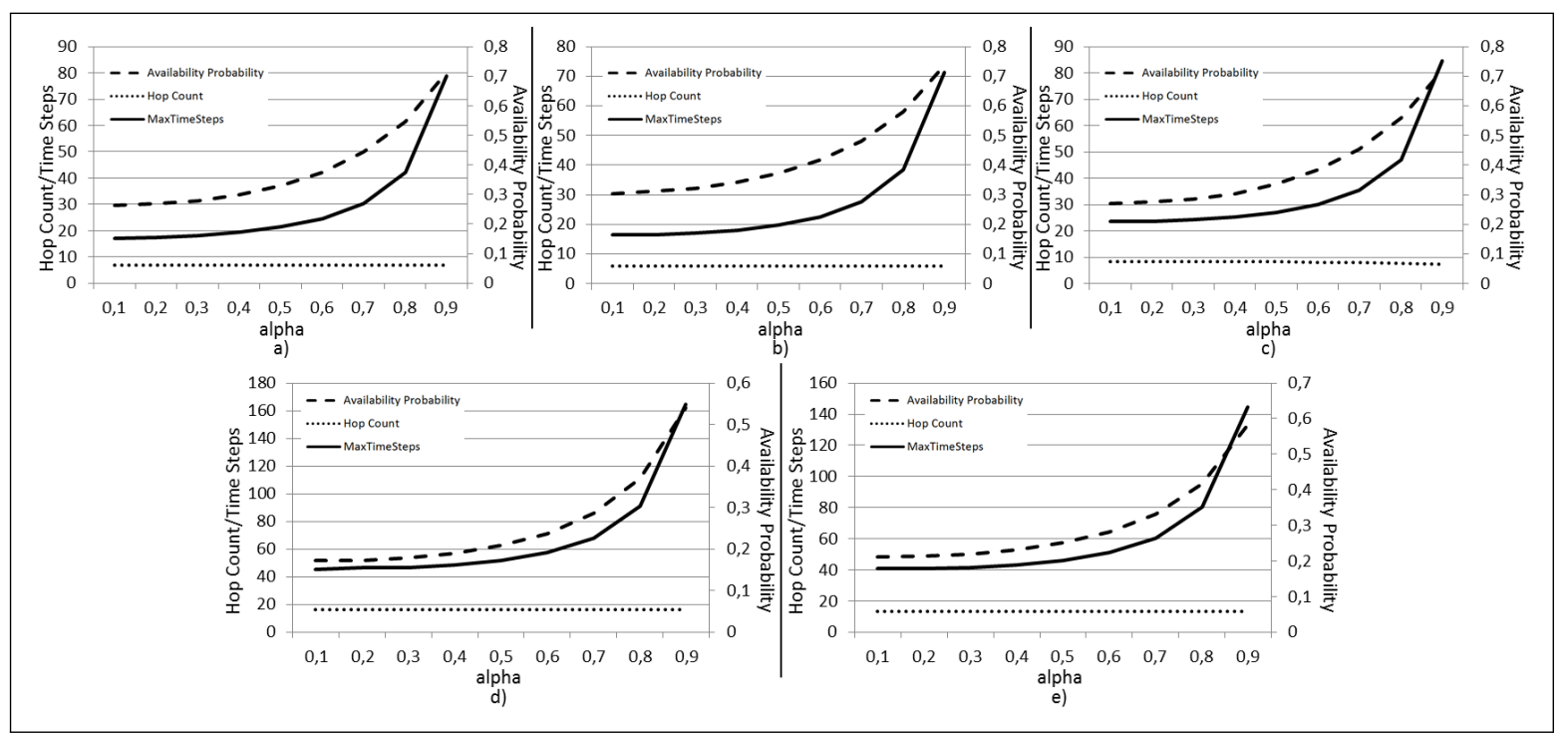

Figure 6: Results for the scenarios with (a) 6; (b) 7; (c) 8; (d) 9; (e) 10 nodes

\section{Conclusions and Future Work}

We have proposed a mathematical model for a Delay/Disruption Tolerant Network in a rural application based on the dynamic availability probabilities of the links between neighboring nodes. These availability probabilities model the movement of nodes and the opportunistic behavior of the solution. We used a linear approximation of the objective function through a logarithmic function that allows a faster implementation and solution.

For an application that can be implemented in a real scenario, we presented a distributed heuristic algorithm where nodes only have access to local information to make decisions. This heuristic was simulated for all the scenarios and it was able to deliver all the messages. The performance of the heuristic regarding the value of the alpha parameter was evaluated. From the results it can be seen that an alpha between 0.6 and 0.8 must be chosen in order to achieve a better availability probability of the paths without a notorious increase in the time needed to deliver all the packets. The hop count is independent of the alpha chosen, it is related to the number of nodes in the network but it also depends on the geometry of the graph and each node's degree.

The DTN implementation proposed here can be used in a rural environment to give Internet connectivity to the people living there using any transportation mean available to them. We have shown that the distributed algorithm used to decide whether to forward or store a message works as expected and can deliver all messages in the scenarios tested; however, this algorithm can benefit from recording previous contacts between nodes and sharing this information with other nodes to create a historical record of contacts and choose the nodes that are most likely to deliver the message in short time.

For future work we are working with the non-lineal model and the subproblem of path generation to evaluate those paths in the master problem (i.e., find the optimal path based on the availability probabilities of the links in it). 


\section{Acknowledgments}

The research leading to these results has received funding from the European Union Seventh Framework Programme (FP7/2007-2013) under grant agreement no. 269985. The author Carlos Velásquez-Villada received funding by the Colombian "Departamento Administrativo de Ciencia, Tecnología e Innovación - Colciencias Conv. 528/2011".

\section{Bibliography}

[1] (2013), Measuring the Information Society, International Telecommunication Union. [Online]. Available: http://www.itu.int/en/ITU-D/Statistics/Pages/default.aspx

[2] Cerf, V.; Burleigh, S.; Hooke, A.; Torgerson, L.; Durst, R.; Scott, K.; Fall, K. \& Weiss, H. (2007), Delay-tolerant networking architecture, RFC4838, April, 1-35.

[3] Andrew T. \& Petkov, D. (2003) The need for a systems thinking approach to the planning of rural telecommunications infrastructure, Telecommunications Policy, 27:75-93.

[4] Johnson D. L. \& Roux, K. (2008), Building rural wireless networks: Lessons learnt and future directions, Proceedings of the 2008 ACM workshop on Wireless networks and systems for developing regions. ACM, 17-22.

[5] Ruxwana, N. L.; Herselman, M. E. \& Conradie, D. P. (2010), ICT applications as e-health solutions in rural healthcare in the Eastern Cape Province of South Africa, Health information management journal, Health Information Management Association of Australia, Limited, 39(1):17-26.

[6] De Savigny, D.; Kasale, H.; Mbuya, C. \& Reid, G. (2008), Fixing health systems: linking research, development, systems, and partnerships, IDRC.

[7] Donner, J. (2004), Innovations in mobile-based public health information systems in the developing world: an example from Rwanda, Workshop on mobile technologies and health: benefits and risks.

[8] De Blasio, G. (2008), Urban-rural differences in internet usage, e-commerce, and e-banking: Evidence from Italy, Growth and Change, 39(2): 341-367.

[9] (2013) Care, [Online]. Available: http://www.care.org

[10] Warren, M. (2002), Adoption of ICT in agricultural management in the United Kingdom: the intra-rural digital divide, ZEMEDELSKA EKONOMIKAPRAHA, 48(1), pp. 1-8.

[11] (2013) Fair trade USA, [Online]. Available: http://www.fairtradeusa.org/

[12] Sohoo, S. (2008), ICT initiative of SAARC agriculture centre in the SAARC region, Computer Science and Information Technology. ICCSIT 08. International Conference on. IEEE, 923-929.

[13] Steinfield, C.; LaRose, R.; Chew, H. E. \& Tong, S.T. (2012), Small and medium-sized enterprises in rural business clusters: The relation between ICT adoption and benefits derived from cluster membership, The Information Society, 28(2):110-120. 
[14] Kannabiran, G.; Xavier, M. \& Banumathi, T. (2008), E-governance and ICT enabled rural development in developing countries: critical lessons from RASI project in India, International Journal of Electronic Government Research (IJEGR), 4(3): 1-19.

[15] Cheng, A.; Sinha, A.; Shen, J.; Mouakkad, S.; Joseph, L. \& Mehta, K. (2012), Opportunities for social innovation at the intersection of ICT education and rural supply chains, Global Humanitarian Technology Conference (GHTC), IEEE, 328-335.

[16] Scott, K. (2009) Delay/disruption tolerant networking, LISA09 Invited Talk, USENIX. [Online]. Available: http://static.usenix.org/events/lisa09/stream1/scott.htm

[17] Warthman, F. (2012), Delay- and Disruption-Tolerant Networks (DTNs). A Tutorial. V. 2.0, Interplanetary Internet Special Interest Group, 2012.

[18] Burleigh, S.; Cerf, V.; Durst, R.; Hooke, A.; Rumeau, R.; Scott, K.; Travis, E. \& Weiss, H. (2001), The Interplanetary Internet: The Next Frontier in Mobility, Internet Global Summit, June, 2001.

[19] Durst, R. C.; Feighery, P. D. \& Scott, K. L. (2000), Why not use the standard internet suite for the interplanetary internet, InterPlanetary Internet (IPN) Technical Information.

[20] Fall, K. (2003), A delay-tolerant network architecture for challenged internet, Proceedings of the 2003 conference on Applications, technologies, architectures, and protocols for computer communications, 27-34.

[21] Scott, K. L. \& Burleigh, S. (2007), Bundle protocol specification, RFC5050, November, 1-50.

[22] Akyildiz, I. F.; Akan, O. B.; Chen, C.; Fang, J. \& Su, W. (2003), InterPlaNetary Internet: state-of-the-art and research challenges, Computer Networks, 43(2):75-112.

[23] Khabbaz, M. J.; Assi, C. M. \& Fawaz, W. F. (2012), Disruption-tolerant networking: A comprehensive survey on recent developments and persisting challenges, Communications Surveys \& Tutorials, IEEE, 14(2), 607-640.

[24] Demmer, M. J. (2008), A Delay Tolerant Networking and System Architecture for Developing Regions, $\mathrm{PhD}$ thesis, University of California at Berkeley.

[25] Pentland, A.; Fletcher, R. \& Hasson, A. (2004), Daknet: Rethinking connectivity in developing nations, Computer, 37(1):78-83.

[26] Seth, A.; Kroeker, D.; Zaharia, M.; Guo, S. \& Keshav, S. (2006), Low-cost communication for rural internet kiosks using mechanical backhaul, Proceedings of the 12th annual international conference on Mobile computing and networking, 334-345.

[27] (2013), Technology and Infrastructure for Emerging Regions, University of California at Berkeley. [Online]. Available: http://tier.cs.berkeley.edu/drupal/

[28] Montoya, G. A.; Velasquez-Villada, C. \& Donoso, Y. (2013), Energy Optimization in Mobile Wireless Sensor Networks with Mobile Targets Achieving Efficient Coverage for Critical Applications, International Journal of Computers Communications $\& 3$ Control, 8(2), 247254.

[29] Velasquez-Villada, C. \& Donoso, Y. (2013), Multipath Routing Network Management Protocol for Resilient and Energy Efficient Wireless Sensor Networks, 1st International Conference on Information Technology and Quantitative Management (ITQM), Suzhou, China, May 16-18, 2013, Procedia Computer Science, 17:387-394. 
[30] Montoya, G. A. \& Donoso, Y. (2013), Energy Load Balancing Strategy to Extend Lifetime in Wireless Sensor Networks, 1st International Conference on Information Technology and Quantitative Management (ITQM), Suzhou, China, May 16-18, 2013, Procedia Computer Science, 17:395-402

[31] Velasquez-Villada, C.; Solano, F. \& Donoso, Y. (2014), Opportunistic Forwarding Algorithm for Delay Tolerant Networks in Rural Applications, International Conference on Computers Communications \& Control, Romania, Oradea, Baile Felix, May 6-10, 2014, Abstracts of ICCCC 2014, ISSN 1844-4334. 